\title{
Five-year outcomes of EVO implantable collamer lens implantation for the correction of high myopia and super high myopia
}

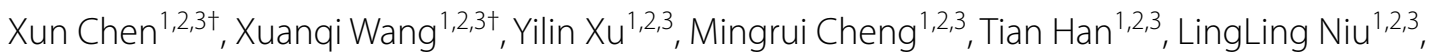 \\ Xiaoying Wang ${ }^{1,2,3^{*}}$ and Xingtao Zhou ${ }^{1,2,3^{*}}$
}

\begin{abstract}
Background: To evaluate the long-term safety, efficacy, predictability, and stability of implantable collamer lens with a central hole (EVO ICL) implantation for correcting high myopia (HM) and super high myopia (SHM).

Methods: This prospective study evaluated 83 eyes of 46 patients who were divided into groups based on their spherical equivalent refractive error (SE): HM group ( $-12 \mathrm{D} \leq \mathrm{SE}<-6 \mathrm{D}$ ) and SHM group (SE $<-12 \mathrm{D})$ ). They were followed up for 5 years after ICL implantation; assessments of uncorrected distance visual acuity (UDVA), corrected distance visual acuity (CDVA), manifest refractive error, axial length, intraocular pressure, corneal endothelial cell density, and vault were conducted, and a questionnaire was administered.

Results: At 5 years postoperatively, the safety indices of the HM and SHM groups were $1.03 \pm 0.10$ and $1.32 \pm 0.39$, and the efficacy indices were $0.83 \pm 0.25$ and $0.86 \pm 0.32$, respectively. In the HM group, $60.47 \%$ and $79.07 \%$ of the eyes were within $\pm 0.50 \mathrm{D}$ and $\pm 1.00 \mathrm{D}$ of the attempted correction, while it was achieved for $22.50 \%$ and $47.50 \%$ of the eyes in the SHM group, respectively. The SE of the HM group decreased from $-9.72 \pm 1.41 \mathrm{D}$ preoperatively to $0.04 \pm 0.39 \mathrm{D} 1$ month postoperatively and $-0.67 \pm 0.57 \mathrm{D} 5$ years postoperatively, while in the SHM group, it decreased from $-15.78 \pm 3.06 \mathrm{D}$ preoperatively to $-0.69 \pm 0.97 \mathrm{D} 1$ month postoperatively and $-1.74 \pm 1.19 \mathrm{D}$ 5 years postoperatively.
\end{abstract}

Conclusion: EVO ICL implantation is safe, effective, and predictable for correcting HM and SHM. CDVA improved more after surgery for SHM, but the growth of axial length still needs attention.

Keywords: Posterior chamber phakic intraocular lens implantation, High myopia, Super high myopia, EVO ICL

\section{Background}

China is a large country with a high prevalence of myopia, and the degree of myopia is generally severe. The proportion of patients with high myopia (HM) and super high myopia (SHM) is higher than that in Western countries

\footnotetext{
*Correspondence: doctxiaoyingwang@163.com; doctzhouxingtao@163.com †Xun Chen and Xuanqi Wang contributed equally and are considered co-first authors

${ }^{1}$ Fudan University Eye Ear Nose and Throat Hospital, No. 19 BaoQing Road, XuHui District, Shanghai 200031, China
}

Full list of author information is available at the end of the article
$[1,2]$. Many doctors and patients are concerned about the correction of low to moderate myopia, either by corneal refractive surgery or implantable collamer lens (ICL) implantation, which has been shown to achieve good visual and refractive results [3-5]. Much attention should be given to patients with HM and SHM because they lack self-confidence, and their choice of professions is limited. Therefore, surgery allows both a correction of the refractive defect and a significant change in personality, career, and lifestyle. 
In the correction of HM using ICL implantation, the cornea and its biomechanics are not affected. The correction range is wide and does not involve limiting the corneal thickness; therefore, it has been widely used in clinical practice [5-7]. Since the advent of clinical application of EVO ICL, several short-term studies [8-11] have confirmed its safety, efficacy, predictability, and stability in correcting HM. However, doctors and patients are concerned about its long-term safety, efficacy, and stability. To the best of our knowledge, there are no reports on the long-term comparisons of EVO ICL implantation used for the correction of HM and SHM. SHM is often accompanied by posterior staphyloma, elongation in axial length, and the presence of myopic maculopathy $[12,13]$. Therefore, this study is based on the hypothesis that the postoperative corrected distance visual acuity (CDVA) improvement space of SHM may be larger than HM because of the poor CDVA preoperatively and that the postoperative axial elongation may be easier. Therefore, this study investigated the safety, efficacy, predictability, and stability of EVO ICL implantation for the correction of HM and SHM for 5 years after surgery to objectively evaluate its long-term visual and refractive outcomes of EVO ICL implantation in the correction of SHM.

\section{Patients and methods}

\section{Patients}

This study adhered to the tenets of the Declaration of Helsinki and was approved by the Ethical Committee Review Board of Fudan University Eye and ENT Hospital. All patients provided written informed consent after the possible risks and benefits of the study were explained.

Patients aged 20-40 years with preoperative spherical equivalent (SE) of $-6.00 \mathrm{D}$ or higher were included in this study. Complications occurring after the surgery, failure to understand the risks of surgery or have unrealistic expectations of surgery outcomes, corneal degeneration, or endothelial cell density $<2000$ cells $/ \mathrm{mm}^{2}$, anterior chamber depth $<2.8 \mathrm{~mm}$, refractive media opacity that severely disturbed vision, a history of autoimmune diseases, and a history of ocular diseases (uveitis, cataract, glaucoma, or retinal detachment) other than myopia and astigmatism were regarded as exclusion criteria.

\section{Pre- and postoperative protocol}

Patients were followed up for five years. Assessments of uncorrected distance visual acuity (UDVA), CDVA, manifest refractive error, axial length (IOL Master, Carl Zeiss, Germany), intraocular pressure (IOP, Tonemeterx-10, Canon, Japan), corneal endothelial cell density (SP-3000P, Topcon Corporation, Japan), and vaults (Pentacam HR, Type 70900; Oculus Optikgeräte GmbH, Wetzlar,
Germany) were conducted. A questionnaire with the following questions was also administered:

1. "Does ICL implantation change your self-image, make you feel more beautiful or more handsome?"

2. "Have you become more confident after ICL implantation?"

3. "Did ICL implantation help you in your career?"

4. "Is life more convenient after ICL implantation?"

5. "If there is another choice, will you choose the ICL implantation again?"

6. "Are you satisfied with ICL implantation as a whole?"

\section{EVO ICL}

The EVO ICL (STAAR Surgical, Switzerland) is a platehaptic single-piece intraocular lens made of collamer. It has a central convex-concave optical zone and incorporates a forward vault to minimize contact with the crystalline lens. A $360 \mu \mathrm{m}$ central hole was included to improve aqueous humor circulation, eliminating the need for preoperative laser peripheral iridotomy. The EVO ICL corrects -0.50 to $-18.00 \mathrm{D}$ myopic spherical refraction and up to $-5.00 \mathrm{D}$ cylindrical refraction. There are four sizes: $12.1 \mathrm{~mm}, 12.6 \mathrm{~mm}, 13.2 \mathrm{~mm}$, and $13.7 \mathrm{~mm}$. Power calculation of the EVO ICL was performed by the manufacturer using a modified vertex formula, according to the provided preoperative refractive parameters. The size of the implanted EVO ICL was determined based on the white-to-white horizontal corneal diameter and anterior chamber depth. $[14,15]$.

\section{Surgical procedure}

All surgeries were performed by experienced surgeons (XW and XZ). The implantation of ICL and the surgical procedures were the same as those used in our previous studies $[14,15]$. During the surgery, a 3-mm temporal corneal incision was made at the temporal corneoscleral limbus. Then, an EVO ICL was inserted into the anterior chamber with an injector cartridge after a viscoelastic surgical agent (1.7\% sodium hyaluronate; Bausch \& Lomb, China) was injected into the anterior chamber to maintain the anterior chamber depth. Sometimes, an additional viscoelastic agent was then placed on the top of the ICL, and an ICL positioning instrument was used to sweep the four haptics of the ICL beneath the iris. Subsequently, a balanced salt solution was used to irrigate the viscoelastic agent. Finally, the surgeon gently pressed the cornea with a cotton swab to close the incision.

\section{Statistical analysis}

This study analyzed six outcomes: (1) change in lines of CDVA from preoperative to postoperative measurements 
(safety); (2) postoperative UDVA compared to preoperative CDVA (efficacy); (3) attempted versus achieved spherical equivalent correction (predictability); (4) changes of SE and axial length; (5) intraocular pressure, vault, and endothelial cell density; (6) questionnaire results.

Statistical analyses were performed using SPSS (version 20.0; SPSS Inc., IBM, USA), and the results are expressed as mean \pm standard deviation (range) values. Kolmogorov-Smirnov test to verify the assumption of normality for the statistical analyses. For continuous variables, summary statistics were analyzed using repeated measures analysis of variance with Bonferroni-adjusted post hoc comparisons and student's t tests as appropriate. For categorical variables, summary statistics were analyzed using chi-square tests. Two-tailed hypothesis testing was performed, and statistical significance was set at $P<0.05$.

\section{Results}

\section{Patient demographics}

A total of 83 eyes of 46 patients (12 men and 34 women) who underwent EVO ICL implantation were included in this study. Their mean age was $28.47 \pm 5.86$ (20 to $40)$ years. The mean preoperative SE was $-12.64 \pm 3.84$ ( -6.12 to -24.50$)$ D. According to the preoperative SE, the patients' eyes were divided into two groups: HM group $(-12 \mathrm{D} \leq \mathrm{SE}<-6 \mathrm{D})$, including 43 eyes (24 patients) and SHM group (SE $<-12 \mathrm{D})$, which included 40 eyes (22 patients). Preoperative data for the two groups are listed in Table 1.

\section{Safety}

No complications were found during the surgery and pupillary block, pigment dispersion, uveitis, glaucoma, cataract, and endothelial cell loss $>30 \%$ were not observed. Macular hemorrhage occurred in one eye and the CDVA recovered after pharmaceutical treatment. Mild anterior subcapsular opacifications were observed in three eyes, which were still under follow-up observation. These cases did not affect the results and statistical analyses. To avoid interference with this study, these cases were excluded when calculating the refractive results.

As shown in Fig. 1, the safety index (postoperative CDVA/preoperative CDVA) of HM and SHM groups were $1.16 \pm 0.16$ and $1.39 \pm 0.37$ at 1 month postoperatively and $1.03 \pm 0.10$ and $1.32 \pm 0.39$ at 5 years postoperatively. There was significant difference between the two groups $(P<0.05)$. The preoperative, 1 month and 5 years postoperative CDVA were $-0.02 \pm 0.04,-0.08 \pm 0.05$, and $-0.03 \pm 0.05 \operatorname{logMAR}$ in the HM group $(P<0.05)$ and $0.12 \pm 0.14,-0.01 \pm 0.10$, and $0.02 \pm 0.08 \log M A R$ in the SHM group $(P<0.05)$, respectively. At 5 years
Table 1 Distribution of preoperative characteristics

\begin{tabular}{llll}
\hline Parameter & HM group & SHM group & P value \\
\hline N, eyes & 43 & 40 & \\
Age (years) & $27.92 \pm 6.09$ & $28.82 \pm 5.61$ & 0.61 \\
Gender (male:female) & $5: 19$ & $7: 15$ & 0.40 \\
UDVA (logMAR) & $1.46 \pm 0.42$ & $1.78 \pm 0.34$ & $<0.001$ \\
CDVA (logMAR) & $-0.02 \pm 0.04$ & $0.12 \pm 0.14$ & $<0.001$ \\
Refractive errors (D) & & & \\
$\quad$ Spherical & $-8.94 \pm 1.34$ & $-14.83 \pm 2.90$ & $<0.001$ \\
Cylindrical & $-1.56 \pm 1.12$ & $-1.90 \pm 0.91$ & 0.13 \\
$\quad$ Spherical equivalent & $-9.72 \pm 1.41$ & $-15.78 \pm 3.06$ & $<0.001$ \\
Keratometric value (D) & & & \\
$\quad$ Flat K & $43.14 \pm 1.44$ & $42.80 \pm 1.47$ & 0.30 \\
$\quad$ Steep K & $44.69 \pm 1.50$ & $44.30 \pm 1.60$ & 0.26 \\
WTW diameter (mm) & $11.90 \pm 0.44$ & $11.92 \pm 0.40$ & 0.83 \\
IOP (mmHg) & $14.27 \pm 2.99$ & $15.36 \pm 3.04$ & 0.10 \\
CCT (mm) & $521.35 \pm 29.55$ & $526.15 \pm 31.24$ & 0.48 \\
Axial length (mm) & $27.08 \pm 0.99$ & $29.68 \pm 1.64$ & $<0.001$ \\
ECD (cells/mm²) & $2689.56 \pm 286.37$ & $2773.89 \pm 206.10$ & 0.14 \\
ICL size (mm) & $13.06 \pm 0.52$ & $13.12 \pm 0.46$ & 0.57 \\
\hline
\end{tabular}

HM high myopia; SHM super high myopia; $N$ number of eyes; UDVA uncorrected distance visual acuity; $C D V A$ corrected distance visual acuity; $D$ diopters; $K$ keratometry; STS sulcus to sulcus; IOP intraocular pressure; $W T W$ horizontal white-to-white diameter; $A C D$ anterior chamber depth; $C C T$ central corneal thickness; $E C D$ corneal endothelial cell density; $I C L$ implantable collamer lens

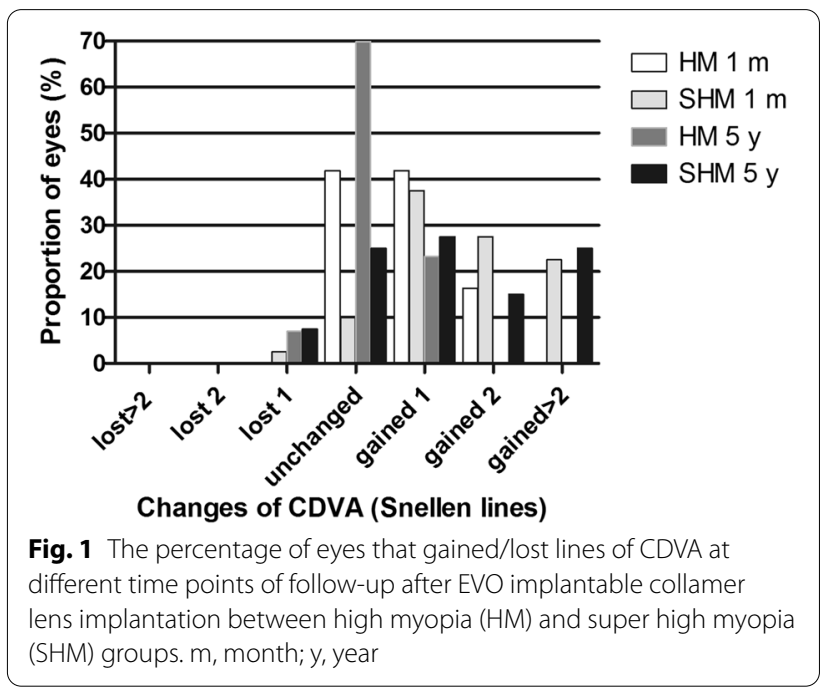

postoperatively, $6.98 \%$ of eyes lost one line of CDVA, $23.26 \%$ of eyes gained one line, and $69.77 \%$ of eyes did not change compared to the baseline in the HM group, and $7.50 \%$ of eyes lost one line of CDVA, $27.50 \%$ of eyes gained one line, $15.00 \%$ of eyes gained two lines, $25.00 \%$ of eyes gained two or more lines, and $25.00 \%$ of eyes did not change compared to the baseline in the SHM group. The percentage of eyes with CDVA 20/20 or better at 
baseline and 1 month and 5 years postoperatively were $95.35 \%, 100.00 \%$ and $100.00 \%$ in the HM group and $40.00 \%, 75.00 \%$, and $67.50 \%$ in the SHM group. All eyes in the HM group had CDVA 20/40 or better at all time points; while $90.00 \%$ of eyes in the SHM group had CDVA 20/40 or better at baseline, this percentage increased to $100.00 \%$ at both postoperative time points.

\section{Efficacy}

As shown in Fig. 2, the efficacy index (postoperative UDVA/preoperative CDVA) of the HM group and SHM group were $1.10 \pm 0.18$ and $1.22 \pm 0.33$ at 1 month postoperatively and $0.83 \pm 0.25$ and $0.86 \pm 0.32$ at 5 years postoperatively, respectively. A significant difference at 1 month $(P<0.05)$ and no significant difference at 5 years postoperatively $(P>0.05)$ were

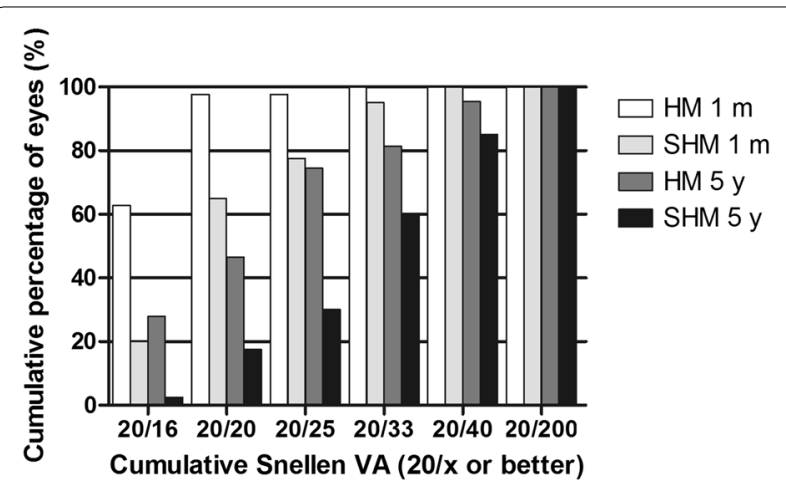

Fig. 2 The cumulative percentage of UDVA at different time points after EVO implantable collamer lens implantation between high myopia (HM) and super high myopia (SHM) groups. m, month; y, year observed between the two groups. The preoperative, 1 month and 5 years postoperative UDVA were $1.46 \pm 0.42,-0.06 \pm 0.07$, and $0.08 \pm 0.15 \log$ MAR in the HM group $(P<0.05)$ and $1.78 \pm 0.34,0.05 \pm 0.12$, and $0.22 \pm 0.15 \operatorname{logMAR}$ in the SHM group $(P<0.05)$, respectively. The percentages of eyes with UDVA 20/20 or better at 1 month and 5 years postoperatively were $97.67 \%$ and $46.51 \%$ in the HM group and $65.00 \%$ and $17.50 \%$ in the SHM group. The percentages of eyes with UDVA $20 / 40$ or better were $100.00 \%$ and $95.35 \%$ in the HM group, $100.00 \%$ and $85.00 \%$ in the SHM group.

\section{Predictability}

A scatter plot with a best-fit line of the attempted versus the achieved spherical equivalent correction is shown in Fig. 3. At 1 month after surgery, the best mimic curve of the HM group was $y=1.01 x+0.16$, with $81.40 \%$ eyes within $\pm 0.50 \mathrm{D}$ and $100.00 \%$ eyes within $\pm 1.00 \mathrm{D}$ of attempted correction value. The best mimic curve of the SHM group was $y=1.04 x$ -0.64 , with $82.50 \%$ eyes within $\pm 0.50 \mathrm{D}$ and $100.00 \%$ eyes within $\pm 1.00 \mathrm{D}$ of attempted correction values. No significant differences were observed between the two groups $(P>0.05)$. At 5 years after surgery, the best mimic curve of the HM group was $y=0.85 x+0.88$, with $60.47 \%$ eyes within $\pm 0.50 \mathrm{D}$ and $79.07 \%$ eyes within $\pm 1.00 \mathrm{D}$ of attempted correction values. Moreover, the best mimic curve of the SHM group was $y=1.03 x-1.54$, with $22.50 \%$ eyes within $\pm 0.50 \mathrm{D}$ and $47.50 \%$ eyes within $\pm 1.00 \mathrm{D}$ of attempted correction
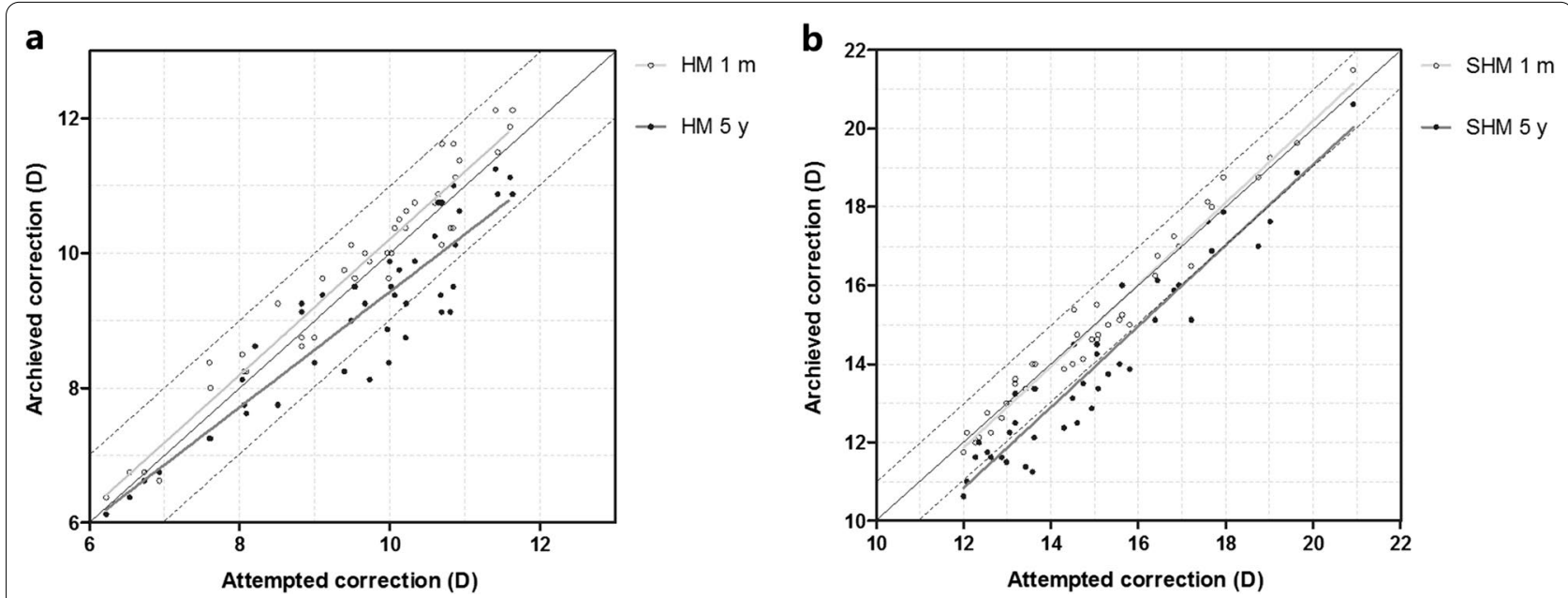

Fig. 3 Scatter plot of attempted versus achieved correction (spherical equivalent) after EVO implantable collamer lens implantation between high myopia (HM) (a) and super high myopia (SHM) (b) groups. The black solid line represents achieved correction=attempted correction, the black dotted line represents achieved correction $=$ attempted correction $\pm 1.00 \mathrm{D}$. m, month; $y$, year 

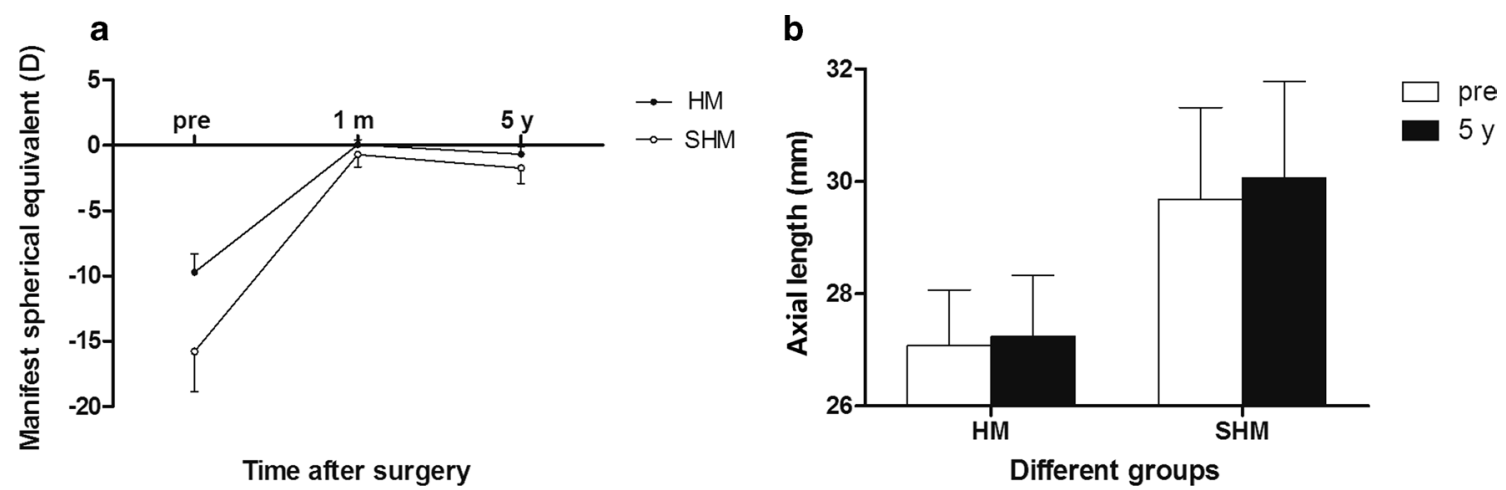

Fig. 4 Manifest spherical equivalent (a) and axial length (b) between high myopia (HM) and super high myopia (SHM) groups after implantation of EVO implantable collamer lens over time. $m$, month; $y$, year; pre, preoperative

values. There were significant differences between the two groups $(P<0.001)$.

\section{Changes of SE and axial length}

The SE of the HM group decreased from $-9.72 \pm 1.41$ $\mathrm{D}$ preoperatively to $0.04 \pm 0.39 \mathrm{D}$ at 1 month and $-0.67 \pm 0.57 \mathrm{D}$ at 5 years postoperatively and from $-15.78 \pm 3.06 \mathrm{D}$ preoperatively to $-0.69 \pm 0.97 \mathrm{D}$ at 1 month and $-1.74 \pm 1.19 \mathrm{D}$ at 5 years postoperatively in the SHM group (Fig. 4a). The differences in SE from 1 month to 5 years postoperatively for the HM and SHM groups were $-0.72 \pm 0.54 \mathrm{D}$ and $-1.05 \pm 0.61 \mathrm{D}$, respectively $(P<0.05)$. The preoperative and 5 year postoperative axial lengths of the HM group were $27.08 \pm 0.99 \mathrm{~mm}$ and $27.24 \pm 1.09 \mathrm{~mm}$, respectively, increasing by $0.16 \pm 0.21 \mathrm{~mm}$. The preoperative and last visit axial lengths of the SHM group were $29.68 \pm 1.63 \mathrm{~mm}$ and $30.07 \pm 1.72 \mathrm{~mm}$, respectively, increasing by $0.39 \pm 0.29 \mathrm{~mm}$ (Fig. 4b). There was a significant difference in axial length growth between the two groups $(P<0.001)$. The differences in SE were related to the axial length growth $(P<0.001)$.

\section{Intraocular pressure, vault, and endothelial cell density}

The preoperative, 1 month and 5 years postoperative IOP of the HM group were $14.27 \pm 2.99(8.3-22.3) \mathrm{mmHg}$, $14.15 \pm 3.47(8.6-23.1) \mathrm{mmHg}$, and $14.58 \pm 2.91$ (11.524.5) $\mathrm{mmHg}$, respectively, and these were $15.37 \pm 3.05$ (10.4-21.3) $\mathrm{mmHg}, 14.81 \pm 2.61(9.9-21.9) \mathrm{mmHg}$, and $15.40 \pm 2.52(10.6-20.0) \mathrm{mmHg}$, respectively, in the SHM group. There was no significant difference in IOP between the two groups at the three time points $(P>0.05$; Fig. 5a).

The 1 month and 5 years postoperative vault of the $\mathrm{HM}$ group were $585.81 \pm 416.53(80-1680) \mu \mathrm{m}$ and $481.16 \pm 415.46(0-1490) \mu \mathrm{m}$, respectively, decreasing by
$104.65 \pm 169.32 \mu \mathrm{m}$. The 1 month and 5 year postoperative vault of the SHM group were $563.75 \pm 323.16$ (130$1420) \mu \mathrm{m}$ and $464.50 \pm 316.31(0-1300) \mu \mathrm{m}$, respectively, decreasing by $99.25 \pm 176.77 \mu \mathrm{m}$. There was no significant difference in vault between the two groups at the two time points after surgery $(P>0.05)$. There was no significant difference in the reduction of vault between the two groups $(P>0.05$; Fig. $5 \mathrm{~b})$.

The preoperative and 5 years postoperative endothelial cell densities of the HM group were $2689.56 \pm 286.37$ $(2092-3213) / \mathrm{mm}^{2}$ and $2591.03 \pm 293.43(1978-3125) /$ $\mathrm{mm}^{2}$, respectively, and the mean loss rate was $3.66 \%$. The preoperative and 5-year postoperative endothelial cell densities of the SHM group were $2773.89 \pm 206.10$ $(2411-3214) / \mathrm{mm}^{2}$ and $2685.11 \pm 213.30$ (2305-3109)/ $\mathrm{mm}^{2}$, respectively, and the mean loss rate was $3.20 \%$. There was no significant difference in endothelial cell densities between the two groups before and 5 years after surgery $(P>0.05$; Fig. $5 c)$.

\section{Questionnaire results}

From Table 2, there was no significant difference in scores between the two groups for any of the questions. It was observed that most HM patients of both groups were more satisfied with their images and more confident with themselves after EVO ICL implantation than before operation. About half of the HM patients thought that the ICL implantation was helpful to their careers. All patients admitted that the ICL implantation had brought convenience to their lives. In particular, the proportion of these changes in patients with SHM was higher.

\section{Discussion}

EVO ICL implantation is a good choice for patients with HM. In this study, the subjects were divided into HM and SHM groups according to SE. UDVA, CDVA, SE, 

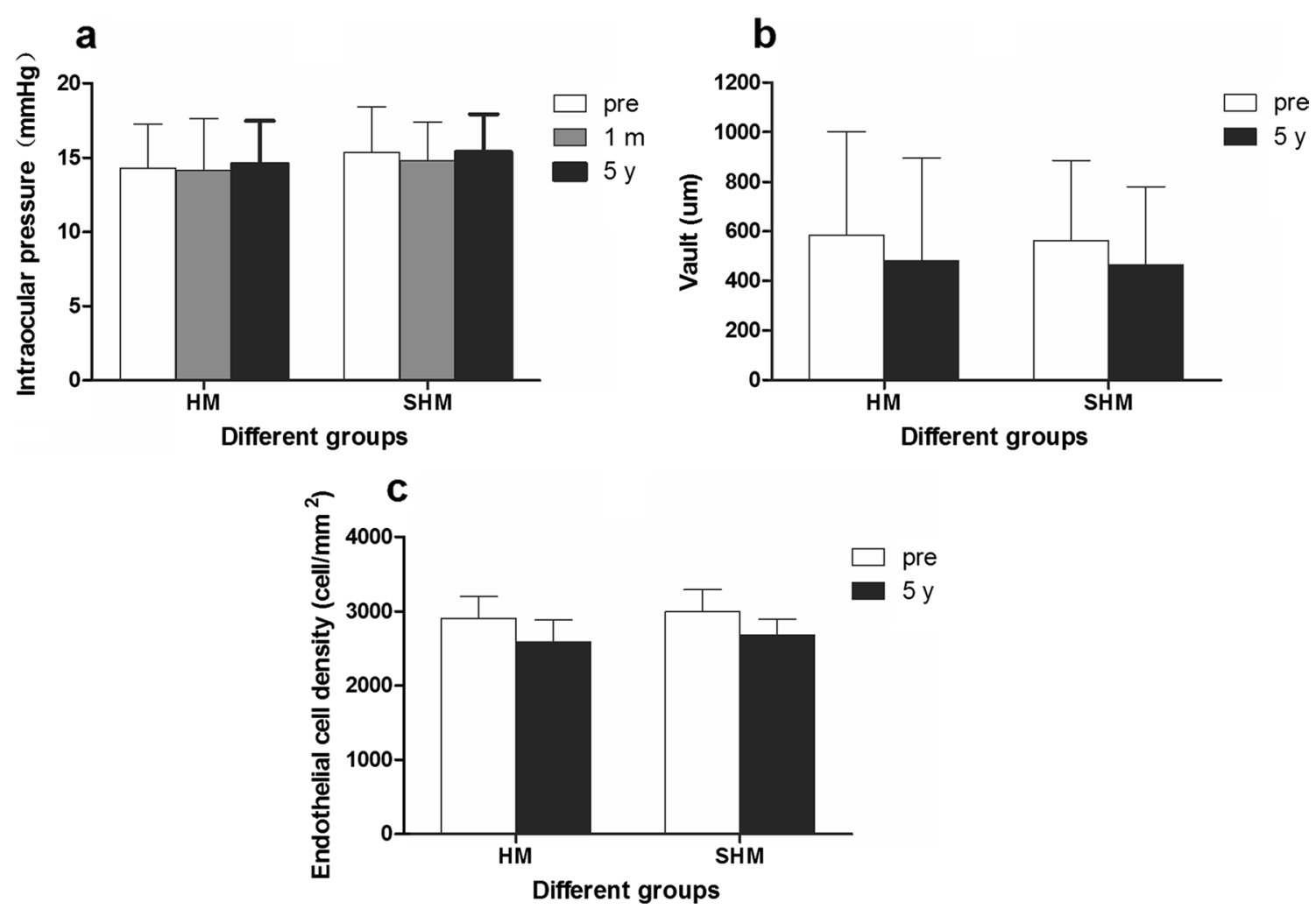

Fig. 5 Intraocular pressure (a), vault (b) and the endothelial cell density (c) between high myopia (HM) and super high myopia (SHM) groups during the period of follow-up after implantation of EVO implantable collamer lens. $m$, month; $y$, year; pre, preoperative

IOP, axial length, endothelial cell density, and vault were measured before and after surgery to evaluate the longterm safety, efficacy, and stability of EVO ICL implantation in the correction of different degrees of HM.

Our study demonstrated that EVO ICL implantation has good long-term safety and efficacy in the correction of HM and SHM. The safety indices of the HM and SHM groups were all above 1.00, from 1 month to 5 years after surgery. The postoperative UDVA and CDVA of the SHM group were worse than those of the HM group, which may be due to the poor preoperative UDVA and

Table 2 Distribution of postoperative questionnaire

\begin{tabular}{lccc}
\hline Question & HM group & SHM group & P value \\
\hline Q1 & $87.50 \%$ & $90.91 \%$ & 0.711 \\
Q2 & $87.50 \%$ & $90.91 \%$ & 0.711 \\
Q3 & $45.83 \%$ & $59.09 \%$ & 0.369 \\
Q4 & $100.00 \%$ & $100.00 \%$ & 1.000 \\
Q5 & $91.67 \%$ & $100.00 \%$ & 0.116 \\
Q6 & $87.50 \%$ & $95.45 \%$ & 0.339 \\
\hline
\end{tabular}

HM high myopia; SHM super high myopia; Q question

Results are expressed as a percentage of the positive answer
CDVA, as well as the severe myopia in the SHM group before surgery. However, the CDVA of the SHM group was more likely to improve because the retinal imaging magnification was higher than that of the HM group. One month after surgery, the efficacy indices of the HM and SHM groups were all above 1.00. However, the efficacy index of the two groups decreased and was lower than 1.00 at 5 years after surgery. The decrease in UDVA and the efficacy index were associated with the refractive change and the axial length change at 5 years after surgery.

Both the HM and SHM groups showed good predictability 1 month after surgery. The predictability results at 5 years after surgery showed a tendency of undercorrection, and the degree of under-correction was more obvious in the SHM group than in the HM group, which was caused by myopic drift, and myopic progression was more severe in SHM. The SE of the SHM group was higher than that of the HM group at 1 month and 5 years postoperatively because some patients had residual SE as their SE exceeded the upper limit of ICL correction. The progression of SE in the HM group and SHM group were $-0.72 \pm 0.54 \mathrm{D}$ and $-1.05 \pm 0.61 \mathrm{D}$, respectively, and the mean axial length growth were $0.16 \pm 0.21 \mathrm{~mm}$ 
and $0.39 \pm 0.29 \mathrm{~mm}$, respectively. There was a correlation between the progression of myopia and axial length growth. Our study showed that the SHM group had more progression of myopia and increased axial length growth. In Kimiya's [16] yearlong study, after the implantation of EVO ICL, the SE progression of low myopia group (mean SE $-4.29 \pm 1.31 \mathrm{D}$ ) and HM group (mean SE $-10.13 \pm 2.64 \mathrm{D})$ were $-0.12 \pm 0.34 \mathrm{D}$ and $-0.18 \pm 0.43$ $\mathrm{D}$, respectively, suggesting that the higher the degree of myopia, the more it progresses. The change in postoperative SE is related to preoperative SE [17]. Therefore, patients with HM, especially those with SHM, should be explained prior to surgery that ICL implantation cannot guarantee the stability of myopia, and there is still the possibility of progression of myopia after surgery. HM, in particular SHM, is basically axial myopia (a myopic refractive state primarily resulting from a greater than normal axial length) [18]. Once the axial length increases, it will lead to the progression of myopia and the decrease of UDVA.

IOP after ICL implantation has also been an area of focus. There was no postoperative IOP increase in either group, indicating that EVO ICL can maintain stable IOP without preoperative iridotomy. Moreover, EVO ICL implantation eliminates the need for Nd:YAG laser iridotomy and reduces the IOP increase caused by pigment dissemination $[19,20]$. In our study, the vault of the two groups showed a decreasing trend in the longterm follow-up of 5 years after surgery, and there was no statistical difference between the two groups. The rate of decrease of vault in the two groups was about $20 \mu \mathrm{m} /$ year, which was similar to those reported in previous studies [21-24]; the vault and its change were independent of the preoperative SE. The mean 5-year endothelial cell loss rates in the HM and SHM groups were 3.66\% and $3.20 \%$, respectively. There was no significant difference between the two groups, which was in line with the physiological endothelial cell loss rule. However, there have been reports of large differences in the rate of endothelial loss [23-27]. The new central port design of the EVO ICL changes the aqueous flow and may therefore influence corneal endothelium cells [28], suggesting that the presence of a central hole does not increase the long-term loss of endothelial cells.

There were no significant differences in the questionnaire scores between the two groups. It can be seen from the results that ICL implantation not only brings a visual correction effect but also improves patients' lives and careers. Most of the patients had better self-images, more confident personalities, and smoother careers than before. For patients with HM and SHM, the value of ICL implantation is not only reflected in visual correction, but also in life.
This study has some limitations, which include the relatively small sample size from a statistical standpoint, lack of a low myopia array, and lack of comparison of visual quality. Besides, this prospective study only had three data points and the rate of missed follow-up in the intermediate period was high because of the Covid-19 situation. This study focused on the 5-year results after surgery, therefore, the data in the intermediate period was not included.

\section{Conclusions}

In summary, our results suggest that EVO ICL implantation is safe, effective, and predictable for correcting HM and SHM. CDVA improved after surgery for the SHM group, but the growth of axial length still needs attention.

\section{Acknowledgements}

Not applicable

\section{Authors' contributions}

The authors were involved in the conception or design of the work, the acquisition, analysis or interpretation of data for the work $(X C, X Q W, Y I X, M R C$, $\mathrm{TH}, \mathrm{LLN}, \mathrm{XYW}, \mathrm{XTZ})$; drafting the work or revising it critically for important intellectual content (XC, XQW); final approval of the version to be published (XC, XQW, YIX, MRC, TH, LLN, XYW, XTZ); agreement to be accountable for all aspects of the work in ensuring that questions related to the accuracy or integrity of any part of the work are appropriately investigated and resolved (XC, XQW, YIX, MRC, TH, LLN, XYW, XTZ). All authors read and approved the final manuscript.

\section{Funding}

This study was supported by National Natural Science Foundation of China (Grant No. 81770955); Joint Research Project of New Frontier Technology in municipal hospitals (Grant No. SHDC12018103); Project of Shanghai Science and Technology (Grant No. 20410710100); Major Clinical Research Project of Shanghai Shenkang Hospital Development Center (Grant No. SHDC2020CR1043B); Project of Shanghai Xuhui District Science and Technology (Grant No. 2020-015); Shanghai Science and Technology Commission (Grant No. 19140900700)

Availability of data and materials

Not applicable.

\section{Declarations}

Ethics approval and consent to participate

This study adhered to the tenets of the Declaration of Helsinki and was approved by the Ethical Committee Review Board of Fudan University Eye and ENT Hospital (2016038).

\section{Consent for publication}

Not applicable.

\section{Competing interests}

The authors declare that they have no competing interests.

\section{Author details}

${ }^{1}$ Fudan University Eye Ear Nose and Throat Hospital, No. 19 BaoQing Road, XuHui District, Shanghai 200031, China. ${ }^{2}$ National Health Commission Key Lab of Myopia (Fudan University), Shanghai, China. ${ }^{3}$ Shanghai Research Center of Ophthalmology and Optometry, Shanghai, China.

Received: 8 April 2021 Accepted: 20 October 2021

Published online: 09 November 2021 


\section{References}

1. Holden BA, Fricke TR, Wilson DA, Jong M, Naidoo KS, Sankaridurg P, et al. Global prevalence of myopia and high myopia and temporal trends from 2000 through 2050. Ophthalmology. 2016;123(5):1036-42.

2. Wang J, Ying GS, Fu X, Zhang R, Meng J, Gu F, et al. Prevalence of myopia and vision impairment in school students in Eastern China. BMC Ophthalmol. 2020;20(1):2.

3. Choi JH, Lim DH, Nam SW, Yang CM, Chung ES, Chung TY. Tenyear clinical outcomes after implantation of a posterior chamber phakic intraocular lens for myopia. J Cataract Refract Surg. 2019;45(11):1555-61.

4. Dishler JG, Slade S, Seifert S, Schallhorn SC. Small-incision lenticule extraction (SMILE) for the correction of myopia with astigmatism: outcomes of the United States Food and Drug Administration premarket approval clinical trial. Ophthalmology. 2020;127(8):1020-34.

5. Siedlecki J, Schmelter V, Mayer WJ, Schworm B, Priglinger SG, Dirisamer $M$, et al. SMILE versus implantable collamer lens implantation for high myopia: a matched comparative study. J Refract Surg. 2020;36(3):150-9.

6. Chen X, Guo L, Han T, Wu L, Wang X, Zhou X. Contralateral eye comparison of the long-term visual quality and stability between implantable collamer lens and laser refractive surgery for myopia. Acta Ophthalmol. 2019;97(3):e471-8.

7. Kim TI, Alió Del Barrio JL, Wilkins M, Cochener B, Ang M. Refractive surgery. Lancet. 2019;393(10185):2085-98.

8. Shimizu K, Kamiya K, Igarashi A, Shiratani T. Early clinical outcomes of implantation of posterior chamber phakic intraocular lens with a central hole (Hole ICL) for moderate to high myopia. Br J Ophthalmol. 2012;96(3):409-12.

9. Lisa C, Naveiras M, Alfonso-Bartolozzi B, Belda-Salmerón L, Montés-Micó $\mathrm{R}$, Alfonso JF. Posterior chamber collagen copolymer phakic intraocular lens with a central hole to correct myopia: one-year follow-up. J Cataract Refract Surg. 2015;41(6):1153-9.

10. Yaşa D, Ürdem U, Ağca A, Yildirim Y, Kepez Yildiz B, Kandemir Beşek N, et al. Early results with a new posterior chamber phakic intraocular lens in patients with high myopia. J Ophthalmol. 2018;2018:1329874.

11. Subudhi P, Patro S, Agarwal P, Khan Z, Subudhi BNR, Mekap C, et al. Safety and efficacy of a new posterior chamber phakic intraocular lens in cases of high myopia: early results. Clin Ophthalmol. 2020;14:3681-9.

12. Ohno-Matsui K. What is the fundamental nature of pathologic myopia? Retina. 2017;37(6):1043-8.

13. Ohno-Matsui K, Lai TY, Lai CC, Cheung CM. Updates of pathologic myopia. Prog Retin Eye Res. 2016:52:156-87.

14. Miao H, Chen $X$, Tian M, Chen Y, Wang X, Zhou X. Refractive outcomes and optical quality after implantation of posterior chamber phakic implantable collamer lens with a central hole (ICL V4C). BMC Ophthalmol. 2018;18(1):141.

15. Chen X, Miao H, Naidu RK, Wang X, Zhou X. Comparison of early changes in and factors affecting vault following posterior chamber phakic implantable collamer lens implantation without and with a central hole (ICL V4 and ICL V4C). BMC Ophthalmol. 2016;16(1):161.

16. Kamiya K, Shimizu K, Igarashi A, Kitazawa Y, Kojima T, Nakamura T, et al. Posterior chamber phakic intraocular lens implantation: comparative, multicentre study in 351 eyes with low-to-moderate or high myopia. Br J Ophthalmol. 2018;102(2):177-81.

17. Gaurisankar ZS, van Rijn GA, Haasnoot GW, Verhoeven VJM, Klaver CCW, Luyten GPM, et al. Long-term longitudinal changes in axial length in the Caucasian myopic and hyperopic population with a phakic intraocular lens. Acta Ophthalmol. 2021;99(4):e562-8.

18. Flitcroft DI, He M, Jonas JB, Jong M, Naidoo K, Ohno-Matsui K, et al. IMIDefining and classifying myopia: a proposed set of standards for clinical and epidemiologic studies. Invest Ophthalmol Vis Sci. 2019;60(3):M20-30.

19. Repplinger B, Kohnen T. Intraocular pressure after implantation of an ICL with aquaport : development of intraocular pressure after implantation of an ICL (model V4c) with aquaport without iridotomy. Ophthalmologe. 2018;115(1):29-33.

20. Kawamorita T, Uozato H, Shimizu K. Fluid dynamics simulation of aqueous humor in a posterior-chamber phakic intraocular lens with a central perforation. Graefes Arch Clin Exp Ophthalmol. 2012;250(6):935-9.

21. Alfonso JF, Fernández-Vega L, Lisa C, Fernandes P, González-Meijome J, Montés-Micó R. Long-term evaluation of the central vault after phakic
Collamer ${ }^{\circledR}$ lens (ICL) implantation using OCT. Graefes Arch Clin Exp Ophthalmol. 2012;250(12):1807-12.

22. Packer M. Meta-analysis and review: effectiveness, safety, and central port design of the intraocular collamer lens. Clin Ophthalmol. 2016:10:1059-77.

23. Shimizu K, Kamiya K, Igarashi A, Kobashi H. Long-term comparison of posterior chamber phakic intraocular lens with and without a central hole (Hole ICL and Conventional ICL) implantation for moderate to high myopia and myopic astigmatism: consort-compliant article. Medicine (Baltimore). 2016;95(14):e3270.

24. Alfonso JF, Fernández-Vega-Cueto L, Alfonso-Bartolozzi B, Montés-Micó R, Fernández-Vega L. Five-year follow-up of correction of myopia: posterior chamber phakic intraocular lens with a central port design. J Refract Surg. 2019;35(3):169-76

25. Kamiya K, Shimizu K, Igarashi A, Ishikawa H. Evaluation of pupil diameter after posterior chamber phakic intraocular lens implantation. Eye (Lond). 2010;24(4):588-94.

26. Lindland $\mathrm{A}$, Heger $\mathrm{H}$, Kugelberg $M$, Zetterström C. Vaulting of myopic and toric implantable collamer lenses during accommodation measured with Visante optical coherence tomography. Ophthalmology. 2010;117(6):1245-50.

27. Lindland $\mathrm{A}$, Heger $\mathrm{H}$, Kugelberg $M$, Zetterström C. Changes in vaulting of myopic and toric implantable collamer lenses in different lighting conditions. Acta Ophthalmol. 2012;90(8):788-91.

28. Yang W, Zhao J, Sun L, Zhao J, Niu L, Wang X, et al. Four-year observation of the changes in corneal endothelium cell density and correlated factors after implantable collamer lens V4C implantation. Br J Ophthalmol. 2021;105(5):625-30.

Ready to submit your research? Choose BMC and benefit from

- fast, convenient online submission

- thorough peer review by experienced researchers in your field

- rapid publication on acceptance

- support for research data, including large and complex data types

- gold Open Access which fosters wider collaboration and increased citations

- maximum visibility for your research: over 100M website views per year

At BMC, research is always in progress.

Learn more biomedcentral.com/submissions 